\title{
Cardiac alterations in cancer-induced cachexia in mice
}

\author{
MIN TIAN ${ }^{1}$, YOSHINORI NISHIJIMA ${ }^{2}$, MICHELLE L. ASP ${ }^{1}$, MICHAEL B. STOUT ${ }^{1}$, \\ PETER J. REISER ${ }^{3}$ and MARTHA A. BELURY ${ }^{1}$ \\ ${ }^{1}$ Department of Human Nutrition, College of Education and Human Ecology, ${ }^{2}$ College of Pharmacy, \\ ${ }^{3}$ Department of Oral Biology, The Ohio State University, Columbus, OH 43210, USA
}

Received February 11, 2010; Accepted March 30, 2010

DOI: 10.3892/ijo_00000683

\begin{abstract}
Cachexia is a common syndrome in advanced cancer patients and causes up to $22 \%$ of cancer-related deaths. It remains elusive whether cancer cachexia causes heart failure. We investigated the effect of cancer cachexia on heart function and cardiac muscle structure in a mouse model. Male CD2F1 mice were inoculated with either colon-26 adenocarcinoma cells (Tumor group) or vehicle (PBS) (No Tumor group and Pair-fed group). Heart function as measured by fractional shortening in vivo using transthoracic echocardiography was performed on day 14 after tumor or PBS inoculation. At necropsy (day 17), hearts were collected for histology, transmission electron microscopy, RT-PCR and SDS-PAGE analysis. Mice from the Tumor group displayed a significantly reduced fractional shortening compared to mice in the No Tumor and Pair-fed groups. In hearts of the Tumor mice compared to the other groups, there was marked fibrosis and transmission electron microscopy revealed disrupted myocardial ultrastructure. Gene expression of troponin I, a regulator of cardiac muscle contraction, was reduced. Moreover, both mRNA and protein levels of myosin heavy chain (MHC) were altered whereby MHC $\alpha$ (adult isoform) was decreased and $\mathrm{MHC} \beta$ (fetal isoform) was increased indicating reactivation of the fetal gene expression pattern. In conclusion, heart function was diminished in mice with tumorinduced cachexia, and this impaired function was associated with increased fibrosis, disrupted myocardial structure and altered composition of contractile proteins of cardiac muscle.
\end{abstract}

\section{Introduction}

Cachexia is a common complication of cancer, refers to the involuntary loss of more than $5 \%$ of body weight within 6 months (1) and may be responsible for as many as $22 \%$ of

Correspondence to: Dr Martha A. Belury, Department of Human Nutrition, College of Education and Human Ecology, The Ohio State University, 1787 Neil Avenue, Columbus, OH 43210, USA

E-mail: belury.1@osu.edu

Key words: echocardiography, myocardium structure, myosin heavy chain cancer deaths (2,3). Cancer cachexia is associated with weakness and fatigue, and it significantly impairs quality of life, response to anticancer therapy and survival $(2,4)$. Cancer cachexia is a syndrome with multiple etiologies including anorexia, systemic inflammation and metabolic dysregulation. The net result is both depletion of adipose tissue depots and skeletal muscle atrophy (5).

While considerable research has addressed the mechanisms of skeletal muscle atrophy in cancer cachexia, the alteration of cardiac muscle structure and metabolism is not well understood. In addition, it remains elusive whether cancer cachexia causes heart failure. A recent study reported that cancer cachexia caused apoptosis-mediated multiple organ failure in rabbits, but the heart was spared from this effect (6). On the contrary, several studies have reported altered metabolism (7) as well as increased protein loss in hearts of patients and mice with cancer cachexia (8-11). Despite a loss of cardiac contractile and myofibrillar proteins, heart performance did not seem to be impaired $(12,13)$. This was attributed to an adaptive response to protect heart function $(14,15)$. An alternative explanation for the loss of cardiac contractile mass is anorexia, which lead to increased degradation and decreased synthesis of protein (16). A limitation to these studies is that heart function was evaluated ex vivo $(7,13,14)$. The purpose of this study was to evaluate heart function in a mouse model of cancer cachexia in vivo, investigate whether functional changes are dependent on reduced food intake and explore whether the changes in function are associated with cellular abnormalities.

\section{Materials and methods}

Experimental animals and design. Five-week-old, male CD2F1 mice (BALB/c x DBA/2; Charles River Laboratories, Wilmington, MA) were housed five per cage at $22 \pm 0.5^{\circ} \mathrm{C}$ on a 12-h light/dark cycle. The mice were fed AIN-93G purified pellet diet (Research Diets, New Brunswick, NJ) containing $7 \%$ fat by weight. When mice weighed approximately $20 \mathrm{~g}$, they were randomly assigned to one of three groups: No Tumor $(n=10)$, Tumor $(n=15)$, or Pair-fed $(n=10)$. On study day 0 , the mice were inoculated subcutaneously in the right flank with either $1 \times 10^{6}$ colon-26 adenocarcinoma cells suspended in $100 \mu 1$ PBS (Tumor group) or vehicle (PBS; No Tumor and Pair-fed groups). Mice in the Pair-fed group were fed the same amount of food consumed by Tumor group. When there 
was approximately $20 \%$ difference in body weight between No Tumor and Tumor groups (day 17 post-inoculation), mice were sacrificed by cervical dislocation. Hearts were harvested first and processed for histological analysis, transmission electron microscopy, and SDS-PAGE. Adipose tissue, skeletal muscles, organs, and tumors were then harvested, weighed, snap-frozen in liquid nitrogen and stored at $-80^{\circ} \mathrm{C}$ until analysis. To obtain more heart tissues with a larger sample size for RT-PCR (real-time-polymerase chain reaction) analysis, a second study was carried out with the same protocol except that there are two groups and larger replicates: Tumor $(n=15)$, No Tumor $(n=15)$. All procedures were in accordance with institution guidelines and approved by the Institutional Animal Care and Use Committee of The Ohio State University.

Echocardiography. At day 14 after tumor/PBS inoculation, in vivo cardiac contractile function was evaluated by transthoracic two-dimensional (2D) and M-mode echocardiography (17). Echocardiography was performed using a GE Vivid7 ultrasound system (GE, Waukesha, WI) with a linear probe (Model i13L; frequency $14 \mathrm{MHz}$ ). Each mouse was anesthetized with $1 \%$ isoflurane and placed in the left lateral decubitus position, and the probe was placed on the left hemithorax. Left ventricular (LV) structure and function were assessed by an evaluation of standard parasternal 2D and M-mode short axis view at the papillary muscle level. All measurements were made in triplicate and averaged by a single investigator (Y. Nishijima). LV end-diastolic (LVIDd) and end-systolic internal diameter (LVIDs) were measured with the American Society of Echocardiography leading-edge method (18). LV fractional shortening (\%) was calculated as (LVIDd-LVIDs)/ LVIDd x 100 .

Histological analysis. Hearts were rapidly excised and a portion of the ventricles was fixed in $10 \%$ neutral-buffered formalin, then paraffin embedded. Sections with a thickness of $5 \mu \mathrm{m}$ were stained with Masson's trichrome, and then analyzed by light microscopy. Fibrosis was independently analyzed in a blinded manner by three evaluators. For each sample, 4-6 microscopic fields were chosen and scored numerically for the extent of fibrosis relative to a positive control.

Transmission electron microscopy. Ventricles from hearts were sliced into $1-\mathrm{mm}^{3}$ cubed pieces and immediately put into fixative ( $2 \%$ glutaraldehyde in $0.1 \mathrm{M}$ phosphate buffer, $\mathrm{pH} 7.4$ ) for $3 \mathrm{~h}$. Sections were post-fixed in $1 \%$ osmium tetroxide for $1 \mathrm{~h}$, dehydrated by an ascending concentrated ethanol series and propylene oxide, followed by embedding in Eponate 12 resin and polymerizing at $60^{\circ} \mathrm{C}$ for $16-24 \mathrm{~h}$. Sections were cut at $80 \mathrm{~nm}$ on a Reichert Ultracut E ultramicrotome, stained with $2 \%$ aqueous uranyl acetate followed by Reynolds lead citrate. Samples were observed in a FEI Tecnai Spirit transmission electron microscope at $80 \mathrm{kV}$.

RT-PCR analysis. RNA was isolated from the ventricles with TRIzol reagent (Invitrogen, Carlsbad, CA) and reverse transcribed with the High Capacity cDNA Archive kit (ABI, Foster City, CA) according to the manufacturer's protocols. RT-PCR analysis was performed with pre-designed primers from Applied Biosystems (TaqMan Gene Expression Assays). Target gene expression was normalized to the endogenous

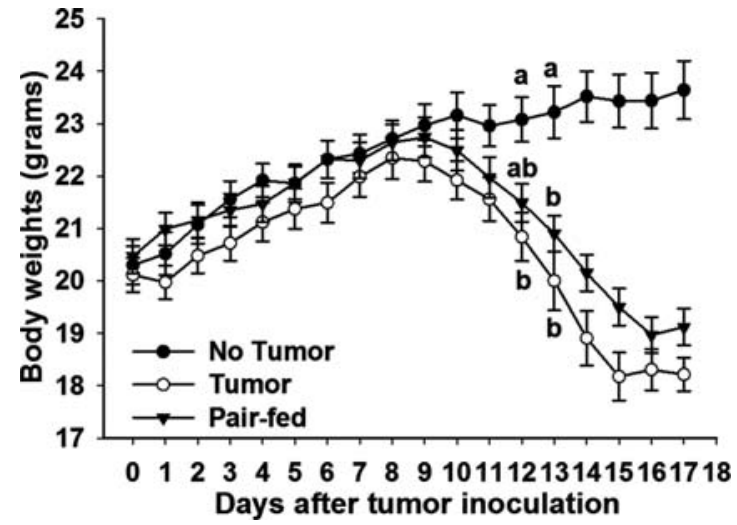

Figure 1. Body weights over time. Male CD2F1 mice were inoculated with either colon-26 adenocarcinoma cells (Tumor $\circ, \mathrm{N}=15$ ) or vehicle (No Tumor $\bullet, N=10$; Pair-fed $\mathbf{\nabla}, \mathrm{N}=10$ ) on study day 0 . Values represent means \pm SEM with significant differences $(\mathrm{P}<0.05)$ denoted by different letters, which indiate the first day such a significant difference was observed, differences were maintained through the completion of the study.

control 18S rRNA amplified in the same reaction and expressed as $2^{-\triangle \triangle C T}$ relative to the No Tumor group (19).

Myosin heavy chain (MHC) isoform separation by SDS-PAGE and silver staining. Heart ventricles ( $20 \mathrm{mg}$ per sample) were homogenized and loaded onto a 7\% SDS-PAGE gel (20). Gels were run at $230 \mathrm{~V}$ for $21 \mathrm{~h}$ at $8^{\circ} \mathrm{C}$, and then silver stained according to methods of Giulian et al (21). The relative amount of $\mathrm{MHC} \alpha$ and $\mathrm{MHC} \beta$ was determined with scanning densitometry as described in Zhong et al (20).

Statistical analysis. Data were analyzed using MINITAB 15 (State College, PA) and are presented as mean \pm standard error of mean (SEM). Differences among Tumor, No Tumor and Pair-fed groups were detected by one-way ANOVA and Tukey's post-hoc analyses. In Figs. 5 and 6, differences between the Tumor and No Tumor groups were analyzed by unpaired, two-sample t-tests. Differences were considered significant at $\mathrm{P}<0.05$.

\section{Results}

Colon-26 adenocarcinoma induces cachexia. The starting body weights among the three groups were not different. There was a 23\% difference in body weight between the Tumor and No Tumor groups at the end of the study (Fig. 1). The No Tumor group showed continual growth throughout the study, as evidenced by continuous body weight increases. The body weight in the Tumor group was significantly lower than the No Tumor group beginning at 12 days after tumor inoculation and this difference continued throughout the rest of the study (Fig. 1). Food intake in Tumor mice was $72 \%$ of that in No Tumor mice (data not shown). Mice in the Pair-fed group demonstrated a growth curve with similar pattern to Tumor mice, despite slightly smaller extent of body weight loss (Fig. 1).

The absolute masses of skeletal muscle and epididymal adipose were significantly less in the Tumor group, compared to the No Tumor group (Table I). In contrast, although the mice of the Pair-fed group lost a similar amount of body 


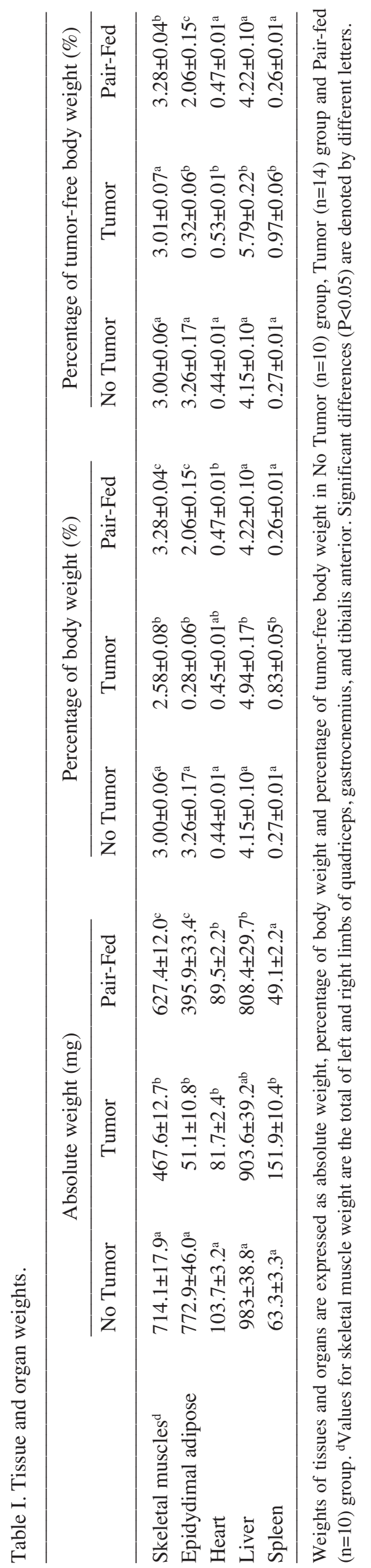

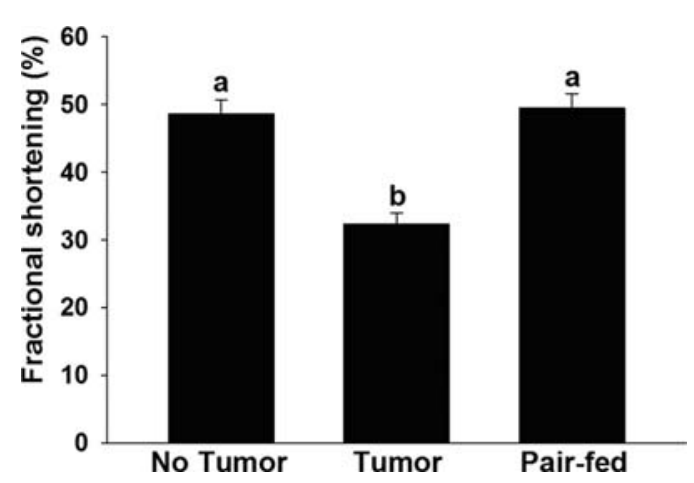

Figure 2. Cardiac contractile function is reduced in mice bearing colon-26 tumors. Fractional shortening measurements by echocardiography 14 days after tumor/PBS inoculation are shown. Values represent means \pm SEM of five mice per group, with significant differences $(\mathrm{P}<0.05)$ denoted by different letters.

Table II. In vivo echocardiographic data.

\begin{tabular}{llcc}
\hline Parameters & No Tumor & Tumor & Pair-Fed \\
\hline Heart rate, bpm & $528 \pm 8^{\mathrm{a}}$ & $418 \pm 13^{\mathrm{b}}$ & $527 \pm 8^{\mathrm{a}}$ \\
LVIDd, mm & $3.4 \pm 0.1$ & $3.3 \pm 0.1$ & $3.2 \pm 0.2$ \\
LVIDs, mm & $1.8 \pm 0.1^{\mathrm{ab}}$ & $2.3 \pm 0.1^{\mathrm{a}}$ & $1.6 \pm 0.1^{\mathrm{b}}$ \\
\hline
\end{tabular}

Values are means \pm SEM. LVIDd, left ventricle $(L V)$ internal diameter at diastole; LVIDs, LV internal diameter at systole. Significant differences $(\mathrm{P}<0.05)$ are denoted by different letters.

weight as the tumor-bearing mice, their muscle weights were markedly higher. When normalized to body weight, the percentages of muscle weight in the Pair-fed mice were higher than that of the No Tumor mice (Table I). These results support that decreased food consumption alone is not sufficient to cause skeletal muscle wasting in this model. Absolute weight of the heart was lower in tumor-bearing mice compared to No Tumor mice, however when normalized to percentage of tumor-free body weight, heart weight was higher in Tumor mice. Both liver and spleen weight were significantly higher in tumor-bearing mice compared to mice in the No Tumor group, regardless of normalization to either percentage of body weight or percentage of tumor-free body weight.

Heart function is impaired in mice with cancer cachexia. To investigate whether cancer cachexia alters cardiac contractile function, echocardiography was performed 14 days after tumor/PBS inoculation, by the time tumor-bearing mice were in the process of losing body weight and there was a $19.6 \%$ difference in body weight between Tumor and No Tumor groups. Echocardiographic assessment revealed that tumorbearing mice displayed a significantly reduced heart rate as well as fractional shortening compared to those in No Tumor and Pair-fed groups (Table II, Fig. 2).

Morphology of cardiac muscle is disrupted in mice with cancer cachexia. Masson's Trichrome staining revealed markedly 


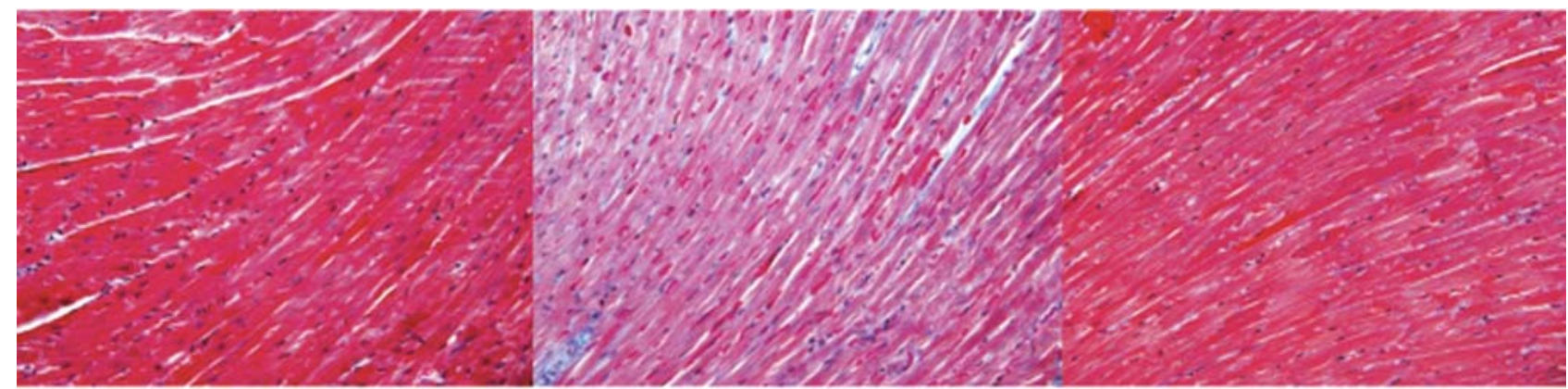

No Tumor

Tumor

Pair-fed

Figure 3. Histological analysis indicates increased fibrosis in hearts (images at $x 40$, Masson's trichrome staining with connective tissue stained blue) of mice bearing colon-26 tumors.

A

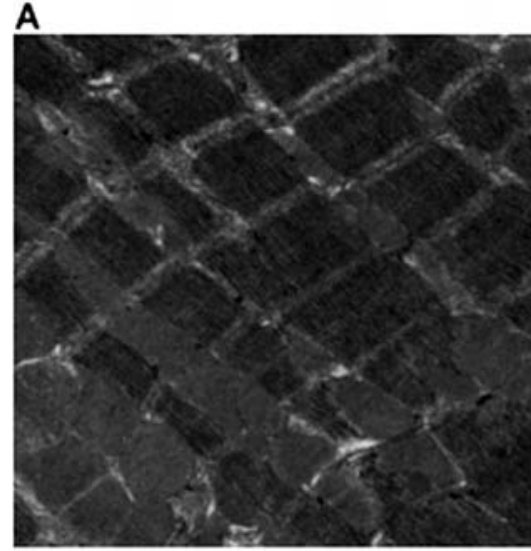

B

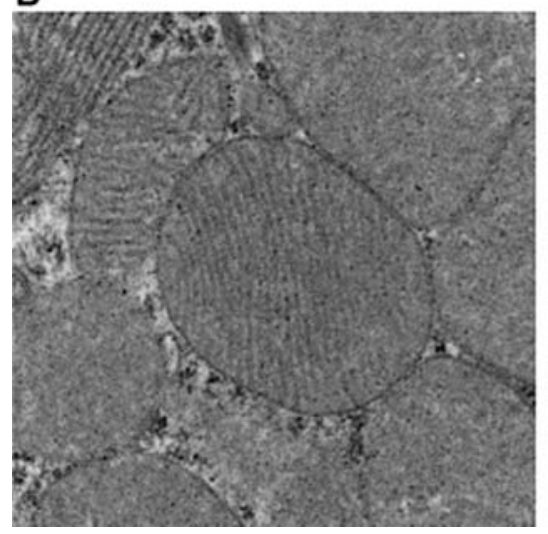

C

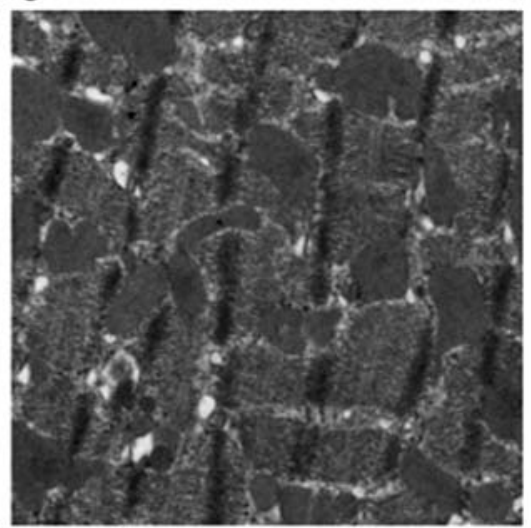

D

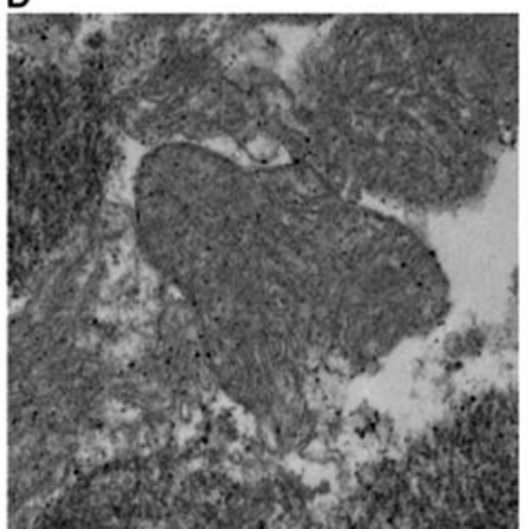

E

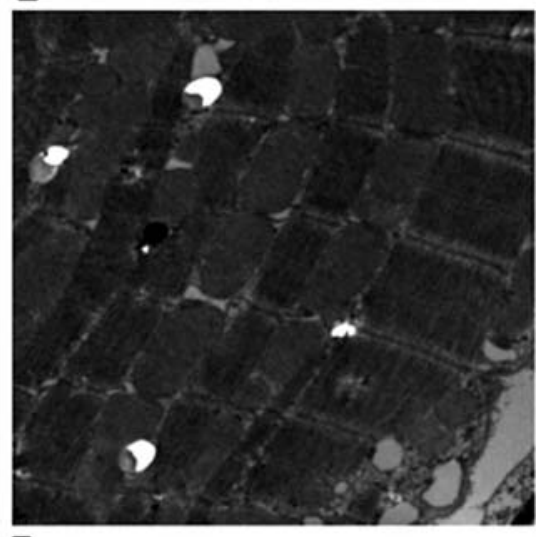

$F$

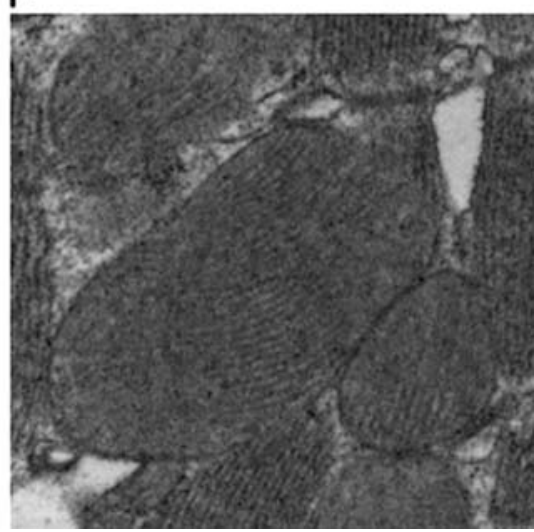

Figure 4. Transmission electron microscopy examination of the myocardial ultrastructure of mice from No Tumor (A and B), Tumor (C and D) and Pair-fed (E and F) groups. Top panels display alignment of sarcomeres and mitochondria, bottom panels are representative pictures of mithochondrial structure. Magnifications are: x13,000 (A, C and E), x50,000 (B), and x49,000 (D and F).

increased fibrosis in the hearts of tumor-bearing mice (Fig. 3). Transmission electron microscopy revealed ultrastructural features of cardiomyocytes from tumor-bearing mice, including disrupted alignments of sarcomeric structure (disorganized I-band, M-band, A-band and Z-line structures) with bundles waving, and randomly dispersed mitochondria with a variety of sizes and shapes. Some mitochondria demonstrated impaired integrity, including broken membranes and disorganized cristea in hearts of tumor-bearing mice (Fig. 4). We were unable to detect any areas with disruptions of sarcomere structure or mitochondria in the No Tumor and Pair-fed groups.
Composition of contractile proteins is altered in cardiac muscle of mice with cancer cachexia. To explore whether the impaired cardiac function and structure is accompanied by altered gene expression of contractile proteins in hearts of tumor-bearing mice, we performed real-time PCR analysis for molecules with potential significance for functional performance. Gene expression of troponin I, a key myofilament protein and regulator of cardiac muscle contractility, was decreased by $38 \%$ in hearts of the Tumor mice compared to the No Tumor group (Fig. 5A). Myosin heavy chain (MHC) isoforms have ATPase activity and are a major determinate 


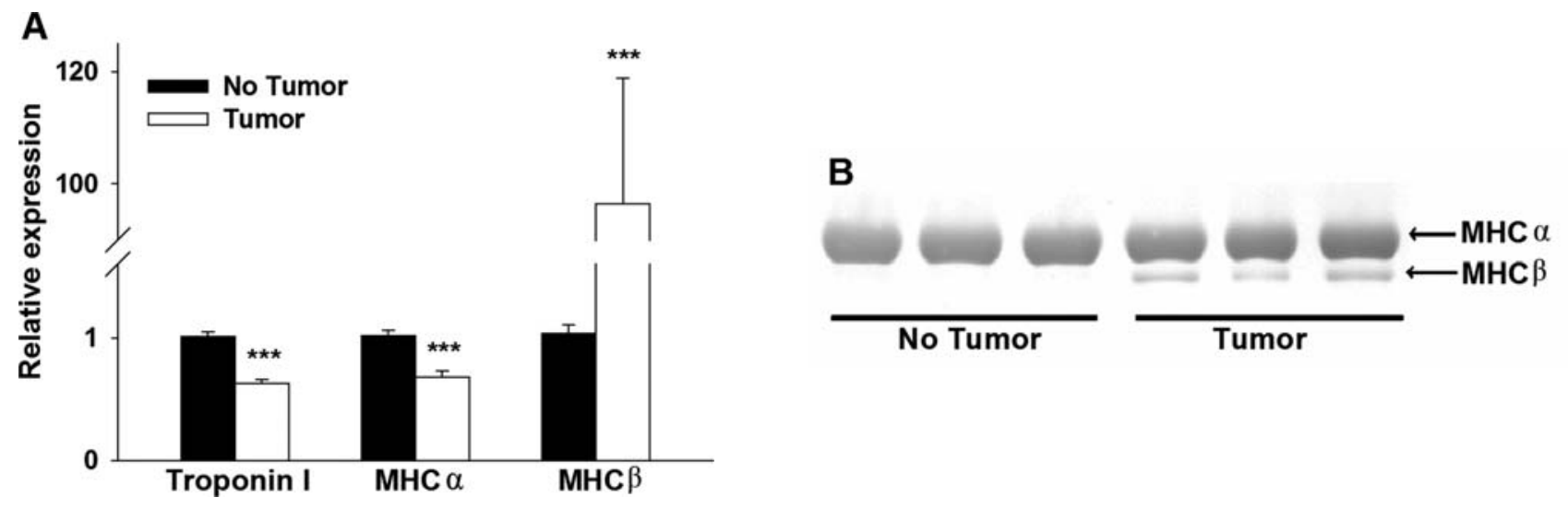

Figure 5. mRNA and protein levels of myofibrillar proteins in hearts of mice in the No Tumor $(n=15)$ and Tumor (n=13) groups. (A) RT-PCR demonstrated decreased gene expression of troponin I, MHC $\alpha$, and increased gene expression of $\mathrm{MHC} \beta .{ }^{* * *} \mathrm{P}<0.001$. (B) Representative myosin heavy chain isoform separation gel reveals increased $\mathrm{MHC \beta}$ protein in tumor-bearing mice $(7.5 \%)$ compared to that in No Tumor mice $(0.4 \%)$.

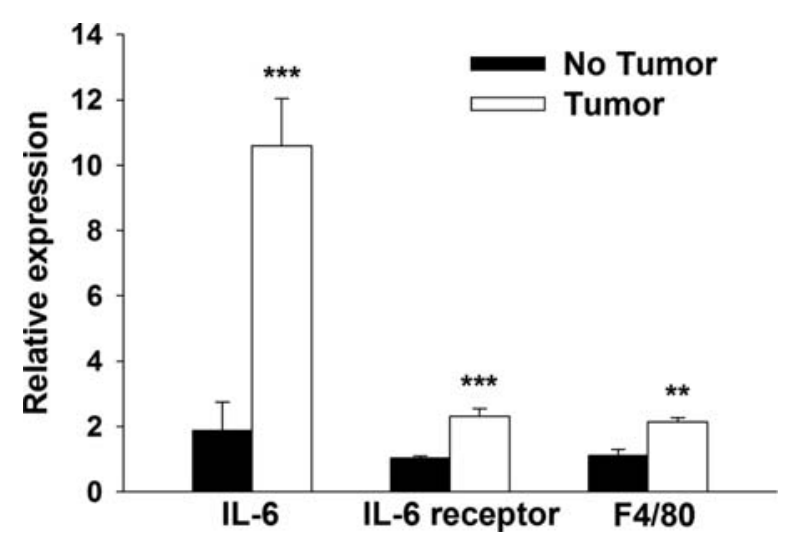

Figure 6. Gene expression of inflammation markers in the hearts of mice in the No Tumor group $(\mathrm{n}=14)$ and Tumor group $(\mathrm{n}=9) .{ }^{* *} \mathrm{P}<0.01,{ }^{* * *} \mathrm{P}<0.001$.

of power generation in muscle. There are two isoforms, $\mathrm{MHC} \alpha$ and $\mathrm{MHC} \beta$ in mammalian hearts, with $\mathrm{MHC} \beta$ predominating during embryonic development and $\mathrm{MHC} \alpha$ predominating after birth in small mammals. Numerous studies suggest that the isoform switch from an 'adult' to 'fetal' isoform (fetal gene pattern reactivation) is associated with heart failure (22-25). Here the mRNA level of MHC $\alpha$ was decreased by $33 \%$, while MHCß was increased by 93 -fold in hearts of Tumor mice compared to the No Tumor group (Fig. 5A). Consistent with the change in mRNA levels, protein levels of $M H C B$ were increased in the hearts of tumor-bearing mice (Fig. 5B).

Markers of inflammation are increased in cardiac muscle of mice with cancer cachexia. Cancer cachexia is characterized by increased levels of inflammation (26). Interleukin-6 (IL-6) plays a critical role in mice with colon-26 adenocarcinoma induced cancer cachexia (27-29). To explore the inflammatory level in the heart, real-time PCR was performed on markers of inflammation. The transcript levels of IL-6 and IL-6 receptors were increased by 5.7-fold and 2.3-fold, respectively in hearts of tumor-bearing mice compared to mice in the No Tumor group. F4/80, a marker of macrophage infiltration, was increased by 1.9 -fold in the hearts of tumor-bearing mice (Fig. 6).

\section{Discussion}

This study evaluated the consequences of cancer cachexia with regard to heart function, myocardium structure, and alterations in composition of myofibrillar proteins. Our results demonstrate that cachectic mice with colon-26 adenocarcinoma tumors exhibited decreased cardiac function, which was not dependent on reduced food intake.

CD2F1 mice inoculated with colon-26 adenocarcinoma are a well-established model of cancer cachexia (27). Cancer cachexia was induced in these mice as shown by significantly lower body weight and loss of adipose and skeletal muscle masses (Table I). These results are consistent with the feature that both muscle and adipose tissues undergo rapid atrophy in this murine model for cancer cachexia $(27,30)$. Both liver and spleen were dramatically enlarged, which is in accordance with the clinical symptoms in colorectal cancer patients that masses of tissues with a high metabolic rate, including liver, spleen and tumor are increased due to systemic inflammation and acute phase protein synthesis (31). While absolute heart weight is lower in tumor-bearing mice compared with mice in the No Tumor group, the proportion of heart relative to tumor-free body weight is higher. The higher relative weight could be attributed to edema (accumulation of fluid and inflammatory cells) considering the increased inflammation status. Inconsistent with the literature $(27,32,33)$ we found food intake is decreased in colon-26 tumor-bearing mice. However, decreased food consumption alone is not sufficient to cause skeletal muscle wasting in this model given the fact that the weights of skeletal muscles were comparable in the Pair-fed and No Tumor groups.

To our knowledge, this is the first study to examine heart function in vivo in conjunction with evaluation of myocardium structure and myofibrillar protein composition in a mouse model of cancer cachexia. A few studies have shown evidence of decreased cardiac muscle mass and loss of contractile and myofibrillar proteins in tumor-bearing animals and cachectic patients $(11,16)$. As for cardiac function, earlier findings suggested that deteriorations in heart function were minimal $(12,13)$. Because of the application of transthoracic echocardiography to study left ventricle function in mice $(35,36)$, 
evaluation of heart function in vivo is accurate and efficient. Our study clearly demonstrated an impaired contractile function in colon-26 tumor-bearing mice, and this functional deterioration was not dependent on reduced food intake.

Sarcomere disorganization and mitochondrial damage are linked with hypertrophic cardiomyopahty (37) and dilated cardiomyopathy (38-40). An earlier study by Sjöström et al (12) reported smaller cross-sectional area and mitochondrial volume density of myocardial cells without severe morphological abnormality in cachectic C57BL/6J mice bearing methylcholanthrene-induced syngeneic sarcoma. In their study, no metabolic markers of myocardial function were found to be altered in tumor-bearing mice, which led the authors to reach the conclusion that myocardial function was protected in cachectic mice (12). In our study, both Masson's trichrome staining and electron microscopy revealed structural changes in the heart of tumor-bearing mice, including increased fibrosis between myofibers, abnormal alignment of myofibers, irregular mitochondrial shape, size and dispersion, and disrupted mitochondrial structure. These changes may be a mechanism underlying the impaired contractile function detected by echocardiography. The different findings from the study of Sjöström et al (12) may be explained by different models as well as the stages of cancer cachexia that were studied. Whereas the former study was carried out at an early stage of cancer cachexia (tumor weight occupied 6-7\% of carcass weight), our study was performed in terminally ill mice. These discoveries mirror a recent comment that heart failure is a slow process in cancer cachexia and may be prominent before death (6).

Cardiac failure is characterized by a switch of gene expression from 'adult' to 'fetal' isoforms in both animals and human patients (22-25). MHC $\alpha$, which predominates in adult mouse hearts has higher ATPase activity than MHCß, which predominates during embryonic development. Even a small magnitude of isoform shift could cause consequences in contractile functions (25). Our RT-PCR results demonstrated significantly decreased MHC $\alpha$ and increased MHCß. At the protein level, these changes could significantly impact heart function (41). Using gel electrophoresis, we were able to confirm the induction of $\mathrm{MHC} \beta$ at the protein level. These changes in MHC composition might be an adaptive response of the heart to save energy, and to a certain extent, contribute to the diminished contractile function in tumor-bearing mice.

Although $\mathrm{TNF} \alpha$ is considered one of the major inflammatory mediators of human cancer cachexia and is present in some animal models of cancer cachexia $(10,42,43)$, IL-6 is the prominent pro-inflammatory cytokine that is elevated in the model of colon-26 tumor-bearing mice (27-29). Our RT-PCR data suggest that local inflammation was increased in hearts of Tumor mice, as demonstrated by the induction of IL-6, IL-6 receptor and macrophage marker F4/80. Increased IL-6 might induce the fetal gene expression pattern observed in Fig. 5A, as previous studies reported such evidence in cultured cardiac myocytes $(44,45)$.

Overall, our data provide compelling evidence for heart function deterioration in association with cancer cachexia. This impaired function is associated with myocardium structure derangement, altered composition of myofibrillar proteins and increased inflammation. This study adds a new dimension in the understanding of the pathogenesis and consequences of cancer cachexia. Specifically, the current dogma states that fatigue and weakness in cancer cachexia can be attributed to skeletal muscle wasting. Our results support the idea that insufficient heart performance might also be responsible for the fatigue symptoms, leading to even less movement and exercise, followed by more severe muscle wasting. Therefore, a vicious cycle is developed and contributes to the morbidity of cancer cachexia. Admittedly in the current study, these cardiac abnormalities were found in cachectic mice at late stages of their lives, and it is rare to see such severe situations in clinical settings. However, it is likely that the alteration of cardiac muscle structure and fetal gene expression pattern occur early and precede the exhibited heart function deterioration. Future studies will determine the time course of these events leading to decreased heart function, and elucidate what is driving these observed cardiac abnormalities. Our previous findings suggested dysregulated lipid metabolism might be in part responsible for the ultrastructural changes of cardiac muscle (46). Further research will seek to determine whether blocking these events in early stages of cachexia using nutritional and/or pharmacological agents may prevent heart function deterioration, improve the quality of life and enhance survival for patients suffering from cancer cachexia.

\section{Acknowledgements}

We would like to thank Richard Mentione for his help with transmission electron microscopy, and Sabahattin Bicer for myosin heavy chain protein isoforms separation. This study was supported by the Kennedy Professorship at The Ohio State University and the Ohio Agricultural Research and Development Center.

\section{References}

1. Inui A: Cancer anorexia-cachexia syndrome: current issues in research and management. CA Cancer J Clin 52: 72-91, 2002.

2. Dewys WD, Begg C, Lavin PT, Band PR, Bennett JM, Bertino JR, Cohen MH, Douglass HO Jr, Engstrom PF, Ezdinli EZ, Horton J, Johnson GJ, Moertel CG, Oken MM, Perlia C, Rosenbaum C, Silverstein MN, Skeel RT, Sponzo RW and Tormey DC: Prognostic effect of weight loss prior to chemotherapy in cancer patients. Eastern Cooperative Oncology Group. Am J Med 69: 491-497, 1980.

3. Warren S: The immediate causes of death in cancer. Am J Med Sci 184: 610-615, 1932.

4. Argiles JM, Almendro V, Busquets S and Lopez-Soriano FJ: The pharmacological treatment of cachexia. Curr Drug Targets 5: 265-277, 2004.

5. Toomey D, Redmond HP and Bouchier-Hayes D: Mechanisms mediating cancer cachexia. Cancer 76: 2418-2426, 1995.

6. Fukuda T, Sumi T, Nobeyama H, Yoshida H, Matsumoto Y, Yasui T, Honda $\mathrm{K}$ and Ishiko O: Multiple organ failure of tumorbearing rabbits in cancer cachexia is caused by apoptosis of normal organ cells. Int J Oncol 34: 61-67, 2009.

7. Drott $\mathrm{C}$ and Lundholm K: Glucose uptake and amino acid metabolism in perfused hearts from tumor-bearing rats. J Surg Res 49: 62-68, 1990.

8. Costelli P, Tullio RD, Baccino FM and Melloni E: Activation of $\mathrm{Ca}(2+)$-dependent proteolysis in skeletal muscle and heart in cancer cachexia. Br J Cancer 84: 946-950, 2001.

9. Tessitore L, Costelli P, Bonetti G and Baccino FM: Cancer cachexia, malnutrition, and tissue protein turnover in experimental animals. Arch Biochem Biophys 306: 52-58, 1993.

10. Costelli P, Carbo N, Tessitore L, Bagby GJ, Lopez-Soriano FJ, Argiles JM and Baccino FM: Tumor necrosis factor-alpha mediates changes in tissue protein turnover in a rat cancer cachexia model. J Clin Invest 92: 2783-2789, 1993. 
11. Lundholm K, Edstrom S, Ekman L, Karlberg I, Bylund AC and Schersten T: A comparative study of the influence of malignant tumor on host metabolism in mice and man: evaluation of an experimental model. Cancer 42: 453-461, 1978.

12. Sjöström M, Wretling ML, Karlberg I, Edén E and Lundholm K: Ultrastructural changes and enzyme activities for energy production in hearts concomitant with tumor-associated malnutrition. J Surg Res 42: 304-313, 1987.

13. Drott C, Ekman L, Holm S, Waldenström A and Lundholm K: Effects of tumor-load and malnutrition on myocardial function in the isolated working rat heart J Mol Cell Cardiol 18: 1165-1176, 1986

14. Drott C, Waldenstrom A and Lundholm K: Cardiac sensitivity and responsiveness to beta-adrenergic stimulation in experimental cancer and undernutrition. J Mol Cell Cardiol 19: 675-683, 1987

15. Ransnäs L, Drott C, Lundholm K, Hjalmarson A and Jacobsson B: Effects of malnutrition on rat myocardial beta-adrenergic and muscarinic receptors. Circ Res 64: 949-956, 1989

16. Drott $\mathrm{C}$, Lönnroth $\mathrm{C}$ and Lundholm K: Protein synthesis, myosin ATPase activity and myofibrillar protein composition in hearts from tumour-bearing rats and mice. Biochem J 264: 191-198, 1989.

17. Talukder MA, Kalyanasundaram A, Zuo L, Velayutham M, Nishijima Y, Periasamy M and Zweier JL: Is reduced SERCA2a expression detrimental or beneficial to postischemic cardiac function and injury? Evidence from heterozygous SERCA2a knockout mice. Am J Physiol Heart Circ Physiol 294: H1426-H1434, 2008.

18. Sahn DJ, DeMaria A, Kisslo J and Weyman A: Recommendations regarding quantitation in M-mode echocardiography: results of a survey of echocardiographic measurements. Circulation 58: 1072-1083, 1978.

19. Livak KJ and Schmittgen TD: Analysis of relative gene expression data using real-time quantitative PCR and the 2(-Delta Delta C(T)) method. Methods 25: 402-408, 2001

20. Zhong Y, Reiser PJ and Matlib MA: Gender differences in myosin heavy chain-beta and phosphorylated phospholamban in diabetic rat hearts. Am J Physiol Heart Circ Physiol 285: H2688-H2693, 2003.

21. Giulian GG, Moss RL and Greaser M: Improved methodology for analysis and quantitation of proteins on one-dimensional silver-stained slab gels. Anal Biochem 129: 277-287, 1983.

22. Wellner M, Dechend R, Park JK, Shagdarsuren E, Al-Saadi N, Kirsch T, Gratze P, Schneider W, Meiners S, Fiebeler A, Haller H, Luft FC and Muller DN: Cardiac gene expression profile in rats with terminal heart failure and cachexia. Physiol Genomics 20: 256-267, 2005

23. Razeghi P, Young ME, Alcorn JL, Moravec CS, Frazier OH and Taegtmeyer H: Metabolic gene expression in fetal and failing human heart. Circulation 104: 2923-2931, 2001

24. Gidh-Jain M, Huang B, Jain P, Gick G and El-Sherif N: Alterations in cardiac gene expression during ventricular remodeling following experimental myocardial infarction. J Mol Cell Cardiol 30: 627-637, 1998

25. Nakao K, Minobe W, Roden R, Bristow MR and Leinwand LA: Myosin heavy chain gene expression in human heart failure. $\mathrm{J}$ Clin Invest 100: 2362-2370, 1997.

26. Fearon KC, Voss AC and Hustead DS: Definition of cancer cachexia: effect of weight loss, reduced food intake, and systemic inflammation on functional status and prognosis. Am J Clin Nutr 83: 1345-1350, 2006.

27. Tanaka Y, Eda H, Tanaka T, Udagawa T, Ishikawa T, Horii I, Ishitsuka $\mathrm{H}$, Kataoka $\mathrm{T}$ and Taguchi T: Experimental cancer cachexia induced by transplantable colon 26 adenocarcinoma in mice. Cancer Res 50: 2290-2295, 1990.

28. Strassmann G, Fong M, Kenney JS and Jacob CO: Evidence for the involvement of interleukin 6 in experimental cancer cachexia. J Clin Invest 89: 1681-1684, 1992.

29. Fujiki F, Mukaida N, Hirose K, Ishida H, Harada A, Ohno S, Bluethmann H, Kawakami M, Akiyama M, Sone S and Matsushima K: Prevention of adenocarcinoma colon 26-induced cachexia by interleukin 10 gene transfer. Cancer Res 57: 94-99, 1997.
30. Acharyya S, Ladner KJ, Nelsen LL, Damrauer J, Reiser PJ, Swoap S and Guttridge DC: Cancer cachexia is regulated by selective targeting of skeletal muscle gene products. J Clin Invest 114: 370-378, 2004

31. Lieffers JR, Mourtzakis M, Hall KD, McCargar LJ, Prado CM and Baracos VE: A viscerally driven cachexia syndrome in patients with advanced colorectal cancer: contributions of organ and tumor mass to whole-body energy demands. Am J Clin Nutr 89: 1173-1179, 2009.

32. Zhou W, Jiang ZW, Tian J, Jiang J, Li N and Li JS: Role of NFkappaB and cytokine in experimental cancer cachexia. World J Gastroenterol 9: 1567-1570, 2003.

33. Lazarus DD, Destree AT, Mazzola LM, McCormack TA, Dick LR, Xu B, Huang JQ, Pierce JW, Read MA, Coggins MB, Solomon V, Goldberg AL, Brand SJ and Elliott PJ: A new model of cancer cachexia: contribution of the ubiquitin-proteasome pathway. Am J Physiol 277: E332-E341, 1999.

34. Asp ML, Tian M, Wendel AA and Belury MA: Evidence for the contribution of insulin resistance to the development of cachexia in tumor-bearing mice. Int J Cancer 126: 756-763, 2010.

35. Gardin JM, Siri FM, Kitsis RN, Edwards JG and Leinwand LA: Echocardiographic assessment of left ventricular mass and systolic function in mice. Circ Res 76: 907-914, 1995.

36. Tanaka N, Dalton N, Mao L, Rockman HA, Peterson KL, Gottshall KR, Hunter JJ, Chien KR and Ross J Jr: Transthoracic echocardiography in models of cardiac disease in the mouse. Circulation 94: 1109-1117, 1996.

37. Muraishi A, Kai H, Adachi K, Nishi $\mathrm{H}$ and Imaizumi $\mathrm{T}$ : Malalignment of the sarcomeric filaments in hypertrophic cardiomyopathy with cardiac myosin heavy chain gene mutation. Heart 82: 625-629, 1999

38. Schaper J, Froede R, Hein S, Buck A, Hashizume H, Speiser B, Friedl A and Bleese N: Impairment of the myocardial ultrastructure and changes of the cytoskeleton in dilated cardiomyopathy. Circulation 83: 504-514, 1991.

39. Hein S, Scholz D, Fujitani N, Rennollet H, Brand T, Friedl A and Schaper J: Altered expression of titin and contractile proteins in failing human myocardium. J Mol Cell Cardiol 26: 1291-1306, 1994

40. Li YY, Chen D, Watkins SC and Feldman AM: Mitochondrial abnormalities in tumor necrosis factor-alpha-induced heart failure are associated with impaired DNA repair activity. Circulation 104: 2492-2497, 2001

41. Tardiff JC, Hewett TE, Factor SM, Vikstrom KL, Robbins J and Leinwand LA: Expression of the beta (slow)-isoform of MHC in the adult mouse heart causes dominant-negative functional effects. Am J Physiol Heart Circ Physiol 278: H412-H419, 2000.

42. Cannon T, Couch M, Yin X, Guttridge D, Lai V and Shores C: Comparison of animal models for head and neck cancer cachexia. Laryngoscope 117: 2152-2158, 2007.

43. Graves E, Ramsay E and McCarthy DO: Inhibitors of COX activity preserve muscle mass in mice bearing the Lewis lung carcinoma, but not the B16 melanoma. Res Nurs Health 29: 87-97, 2006.

44. Saito S, Aikawa R, Shiojima I, Nagai R, Yazaki Y and Komuro I: Endothelin-1 induces expression of fetal genes through the interleukin-6 family of cytokines in cardiac myocytes. FEBS Lett 456: 103-107, 1999.

45. Tanaka T, Kanda T, Takahashi T, Saegusa S, Moriya J and Kurabayashi M: Interleukin-6-induced reciprocal expression of SERCA and natriuretic peptides mRNA in cultured rat ventricular myocytes. J Int Med Res 32: 57-61, 2004.

46. Tian M, Asp ML, Wendel AA and Belury MA: Dysregulation of lipid metabolism in cardiac muscle of mice with cachexia. FASEB J 22: 147.5, 2008. 